\title{
Chapter 3: Soft Systems Thinking Approach to e-HRM Project Management
}

\section{Mohan Thite \& NMK Bhatta}

Thite, M. \& NMK Bhatta. (2019). Soft systems thinking approach to e-HRM Project Management. In M. Thite (Ed.). e-HRM: Digital Approaches, Directions \& Applications, Abingdon, UK: Routledge. P. 42-56

\section{Abstract}

Organisations spend enormous amount of money, time and efforts to leverage technology to deliver faster, cheaper and better HR services; however, majority of IT projects fail to finish on time, within budget and satisfy users. This is primarily due to lack of systems perspective and soft skills. This chapter highlights the critical importance of systems thinking approach, systems development life cycle and quality management framework underpinned by people capability maturity model. The accompanying case study provides further evidence of how non-technical factors contribute to project success.

\section{Leaning Objectives}

1. Explain why the failure rate for technology projects is very high and how success factors are mainly around people and process rather than technology

2. Describe the key principles behind systems thinking and the soft skills needed to adopt systems perspective

3. Explain the phases of system development life cycle (SDLC)

4. Discuss the different levels of People Capability Maturity Model (P-CMM) and the human resource aspects to be considered at each level

\section{Introduction}

The Standish Group produces the CHAOS Reports every year judging the success of around 50,000 software development projects world-wide, based on six success criteria, namely, on Time, on Budget, on Target, on Goal, Value and Satisfaction. According to the 2015 report, only $29 \%$ of the projects were considered 'successful', $52 \%$ were considered 'challenged' (over budget and over time) and 19\% ended in 'failure'. For 'grand' projects (costing over $\$ 100$ million in labor costs), the success rate was only $2 \%$; however, small projects reported a success rate of 62\%. (Hastie \& Wojewoda, 2015). 
The report also concluded that for those projects that were successful, the following were the key success factors, in the order of importance:

1. Executive Support: Financial and emotional backing from top management

2. Emotional Maturity: Soft skills, including overcoming the 'five deadly sins' of project management (as referred to in Table 3.1), managing expectations, consensus building, and collaboration

3. User Involvement: Involvement of the users in project decision-making and information-gathering process

4. Optimization: A structured means of improving business effectiveness

5. Skilled Staff: People who understand both the business and the technology

6. Standard Architecture: Integrated practices, services, and products for developing, implementing, and operating software applications

7. Agile Proficiency: The ability of the project team in following the agile process (see next chapter for a detailed explanation of the agile methodology)

8. Modest execution: Having a process with few moving parts, and those parts are automated and streamlined.

9. Project Management Expertise: Application of knowledge, skills, and techniques to project activities in order to meet or exceed stakeholder expectations

10. Clear Business Objectives: The understanding of all stakeholders and participants and project alignment with the organization's goals and strategy

There are two key lessons from the above report: one, despite the enormous amount of money, time and efforts spent on planning, designing, implementing, and maintaining technology projects, the failure rate is alarmingly high. This is particularly so with large scale projects due to their extreme complexity, interdependencies and communication challenges (Brock, Saleh \& Iyer, 2015). Two, technology is secondary in the success of a technology project management. It is the non-technical factors, including the soft skills as mentioned in Table 3.1 that are crucial in the success or failure of technology projects (Martinsons \& Chong, 1999).

Insert Table 3.1 somewhere here 
Accordingly, organizations need to adopt a long-term, holistic, system thinking approach to designing and delivering technology projects. Technical projects are primarily resource intensive and the resources involved are knowledge workers with unique characteristics and difficult to handle attitudes. An in-depth knowledge of how technology projects are managed and what factors lead to their success or failure would help human resource (HR) professionals to provide appropriate customised support to the technical teams throughout the project phases.

In this chapter, we will explain the basics of systems thinking, the Systems Development Life Cycle (SDLC) that underpins systems thinking and role of HR in each phase of the SDLC. We will then introduce People Capability Maturity Model (P-CMM), a quality management framework widely used in the IT projects with associated HR aspects. Finally, using a Case Study, we will summarize the key managerial lessons to be learnt to increase the chances of success in e-HRM projects.

\section{Systems Thinking}

A popular adage attributed to Aristotle says, 'the whole is more than a sum of its parts'. It refers to the capability of individuals coming together to achieve more than what they could do on their own, 'provided' they act together as a unified system. It is also reflected in another popular phrase, 'united we stand, divided we fall'. When we apply this thinking to complex organisational systems, such as technology projects, we can surmise that if the individuals involved in the system focus only on themselves or the tasks they are performing without seeing the 'big picture', they fail to recognise what others are doing or thinking and tend to blame each other when things start falling apart.

A system is "any group of interacting, interrelated, or interdependent parts that form a complex and unified whole that has a specific purpose" (Kim, 1999, p. 2). According to Arnold \& Wade (2015, p. 675), "systems thinking is a set of synergistic analytic skills used to improve the capability of identifying and understanding systems, predicting their behaviors, and devising modifications to them in order to produce desired effects. These skills work together as a system”.

Squires, Wade, Dominick, \& Gelosh (2011) explain further that "systems thinking is the ability to think abstractly in order to

- incorporate multiple perspectives; 
- work within a space where the boundary or scope of problem or system may be "fuzzy";

- understand diverse operational contexts of the system;

-identify inter- and intrarelationships and dependencies;

- understand complex system behavior; and most important of all

-reliably predict the impact of change to the system".

These skills are however hard to acquire. As Senge (1990, p. 7) explains, human endeavours are also systems bound by invisible interrelated actions and people inside the system tend to focus on 'snapshots of isolated parts of the system' and find it 'hard to see the whole pattern of change'.

The project management discipline incorporated 'systems engineering' thinking in the 1990s. Sankaran, Haslett \& Sheffield (2010) note that "As project management spread its wings, "softer" or more people-centric issues, such as personnel management, motivation, team performance, team structure, stakeholder management, negotiation, communications management, and leadership, were added to the list of Best Practices (McConnell, 1996). Systems thinking approaches also started to move away from "hard systems" (product and technology-centric) to "soft systems" (people and process) approaches (McConnell, 1996, p. $11)$.

The critical importance of people and process over product and technology in project management places HR managers as a key driver to ensure e-HRM project success. Any job advertisement for Human Resource Information Systems (HRIS) manager underscores the fundamental role of the position as a bridge between HR and IT professionals to ensure they understand each other's role and work together as a team. Green (2017) explains that as part of the job description of an HRIS manager

"Strong analytical, troubleshooting and problem-solving skills are integral to the competence of HRIS managers. When employee management software crashes, for example, the manager uses these skills to troubleshoot the software and implement appropriate solutions, as well as uses operating systems and programming tools effectively. HRIS managers also need strong leadership skills to guide and supervise the HRIS team, which may include database administrators and data analysts. Good communication skills are also 
important to these managers, because they need to write clear and concise reports and communicate effectively with vendors of information systems".

If there is no proper understanding between the users, business analysts and software programmers of the project goals and requirements, the final product may fail to meet the user expectations, and result in terrible loss of time, efforts and money.

\section{System Development Life Cycle Using Waterfall Methodology}

Traditionally, Software projects followed a development approach Called "Waterfall Methodology". This approach was originally promoted by the US Department of Defence in the 1950s and because of rugged delivery capability its prominence continues even today. Waterfall emphasizes the concept of serially phased development philosophy in which various project phases are carried out in a serial manner. Every phase is completed with proper Verification and Validation process and detailed documentation. Once completed, a phase is considered frozen and never revisited except under extraordinary circumstances. The phases of the waterfall model formed the System Development Life Cycle (SDLC) for IT Projects. Figure 3.1 depicts various phases of SDLC.

Insert Figure 3.1 somewhere here

We explain below each phase of SDLC.

\section{Project Planning}

The Systems Development Life Cycle (SDLC) starts with the objective to align the project as close to the strategic business goals of the organisation as possible. Clear priorities need to be established on how best to leverage technology to meet organisational goals and derive competitive advantage. This requires clear alignment between organisational strategy, information technology (IT) strategy and human resources (HR) strategy. For instance, if the organisational strategy is to be a global technology leader, the IT strategy would be to deploy and harness state of the art, future-focused technologies. Similarly, the HR strategy would be 
to acquire best of breed applicant tracking system (ATS) to attract best talent and learning management system (LMS) to train the workforce.

The planning phase includes both long-term strategic planning and short-term operational planning. During this phase, HR needs to prioritise which HR technology projects are critical and how best to implement them.

\section{Requirement Analysis}

The next phase of the SDLC deals with detailed requirement gathering to prepare a project scope document (typically called Software Requirement Specification or SRS). It collects information about the current and proposed system in order to answer the three crucial questions, namely, where are we, where we want to go and how do we get there. To answer these critical questions, the organization needs to document its current capabilities and future needs in order to clearly define the scope of an e-HRM project.

For example, for a e-recruitment project, the analysis team, consisting of HRIS manager, key users from concerned departments and external consultants, needs to determine which parts of the recruitment system need be automated, how does it interface with other HR and management systems, what information should be included, how does the information flow through the system, what types of reports would be generated, would the system be cost effective and generate reasonable return on investment, what impact it would have on staffing and how it would ensure information security and privacy.

\section{Design}

The next phase deals with the design of appropriate IT solution, including hardware, software, and network infrastructure, which is essentially a technology intensive activity involving technical architects and designers. Once the blueprint of the new system is prepared, the project team prepares a request for proposal (RFP) which is used to identify, assess and finalise a vendor who can provide and implement the system. The most common approach here is to buy a commercial off the shelf (COTS) product from a reputed vendor with established credentials and track record.

\section{Implementation}


The implementation phase of the SDLC follows the design phase. This is the longest phase of the entire SDLC and usually takes up to $60 \%$ of the project schedule. During this phase the system is coded and tested before going 'live'. While the activity is essentially technical in nature, HR needs to be actively involved at every stage during the implementation phase. People's motivation levels are to be maintained at the highest level to surmount every challenge that comes their way during this phase. HR personnel need to integrate themselves with the project team and be present on a continuous basis to sense signals of stress and disengagement so that they can initiate necessary action before it snowballs into a major breakdown. Routine stress relief events such as outdoor fun activities which improve emotional bonding among people would be a major responsibility for HR in this phase. For these activities to be effective, HR teams must be co-located with the project teams to give them a feeling that HR is one among them.

During the implementation phase, managing employee attrition is another challenge that HR has to deal with. While attrition may not be completely avoidable as people with critical IT skills are always in high demand, strategic management of employee attrition is essential. For instance, every time a performance appraisal cycle is completed, there will be emotional stress among people whose performance rating doesn't match with their expectations resulting in unexpected resignations. HR needs to handle each case sensitively and proactively.

\section{Project Closure}

The next phase in SDLC deals with the closure of the project. Project closure can be sometimes most frustrating as many of the so called less important activities postponed during various other phases become a serious bottleneck in project closure. Most of the technical personnel will be looking for their next assignment. The project manager finds it extremely challenging to wrap up all pending activities. A major responsibility of the HR during this phase will be to keep the team together and make them work towards the project closure in a focussed manner.

\section{Maintenance \& Support}

Maintenance and support phase, often called the 'forgotten phase', follows the project closure. It includes corrective maintenance (where bugs in the system are fixed), adaptive maintenance (where modifications and enhancements are carried out), perfective maintenance 
aimed at continuous improvement and preventative maintenance to fine tune the hardware and software. Since most of the maintenance projects are subject to Service Level Agreement (SLA) with outside third-party service providers, the promptness with which the vendor responds and resolves the maintenance issues is critical. Therefore, HR needs to acquire the capability for effective vendor management.

Figure 3.2 summarises the key technical and human resource aspects and success factors of the each phase of SDLC.

Insert Figure 3.2 somewhere here

As highlighted in Figure 3.3, there are two critical success factors that exert a major influence on all aspects of SDLC, namely, user involvement throughout the process and ongoing and integrated communication with diverse stakeholders. Hsu, Liang, Wu, Klein \& Jiang (2011, p. 514) emphasise that "active and fruitful participation of users in the development projects of information systems (IS) is a crucial factor in achieving success. However, difficulties such as conflict and apathy often plague IS development projects that do not rely on building effective partnerships between users and those responsible for the development". Thus, most problems in software projects occur due to the neglect of social aspects, such as stakeholder resistance (Vrhoves, Hovelja, Vavpotic \& Krisper, 2015).

\section{Evaluation of SDLC/Waterfall Model}

As the analogy of waterfall suggests, it is a linear, structured and sequential model where each phase must be fully implemented before embarking on the next phase and unless exceptional circumstances warrant, the previous phases are not revisited.

The advantages of this model are that

- It adopts the systems thinking perspective where all phases from inception to completion are well thought out, planned and executed

- It is easier to understand and use

- Since each phase is well defined and the project moves to the next stage only after ensuring successful completion of each phase as measured by clearly defined deliverables, tight project control and discipline are established 
- It is suitable for well-defined large-scale projects with clear project goals, time lines, requirements and processes that are well communicated to all stakeholders.

However, the disadvantages of the model are that

- There is no margin for errors and leaving little scope to make major changes to the project directions, requirements and deliverables, no matter how well justified the changes are.

- Strict testing and sign-off of each phase before moving to the next stage may cause undue delays

- Once a phase is completed there is no going back even though subsequent changes to the model incorporate iterative capability

- One cannot see the working version of the application until the end phase is reached

- Not suitable for fast changing and uncertain business environments where flexibility and adaptability are the key.

\section{Quality Management Framework: People Capability Maturity Model}

Human resources are the backbone of any organization in the $21^{\text {st }}$ century knowledge economy. The knowledge workers are extremely critical to the success of high-technology firms in the information and communication technology (ICT) industry where an optimum combination of people, process and technology provide sustainable competitive advantage. However, the industry has traditionally suffered from serious people management issues, such as high turnover, burnout, stressful work environment and critical skill shortages. It took quite some time for the industry to realise that no matter how great the technology that was used, the key challenges were process and people related, as highlighted throughout this chapter.

To ensure sustainability, repeatability and scalability without compromising on quality, particularly while handling large scale software projects and to address critical people issues, the Software Engineering Institute (SEI) at Carnegie Mellon University developed a process maturity framework called People Capability Maturity Model (P-CMM) as a model of best practices for managing and developing an organization's workforce. Today it is widely used by the most major ICT services firms and those that achieve P-CMM Level 5 maturity level are highly regarded. 
According to Curtis, Hefley \& Miller (2009, p. xiii) "the People CMM helps organizations characterize the maturity of their workforce practices, establish a program of continuous workforce development, set priorities for improvement actions, integrate workforce development with process improvement, and establish a culture of excellence". P-CMM consists of five maturity levels (as depicted in Figure 3.3) that establish successive foundations for continuously improving individual competencies.

Insert Figure 3.3 somewhere here

As explained by Curtis, Hefley \& Miller (2009, p. 12-13),

Level 1 (Initial: Inconsistent Management): At this basic level, organisations are characterised by ad hoc and inconsistent work processes and the project personnel resort to cutting corners in a rush to meet deadlines resulting in poor quality, delays and cost blowout. "A fundamental premise of the process maturity framework is that a practice cannot be improved if it cannot be repeated" (p. 12). Due to the immaturity of the organisation, people struggle to repeat successful work practices and meet delivery deadlines as goalposts keep changing. In this environment, people routinely deflect responsibility and become emotionally drained and detached. Managers either ignore or ill-equipped to deal with HR issues leading to high employee turnover and low morale. The case study firm described at the end of this chapter typifies Level 1 organisations.

Level 2 (Managed: People Management): In order to escape from inconsistent and unproductive work practices, organisations must first establish a stable work environment. "The primary objective of a Maturity Level 2 environment is to enable people to repeat practices they have used successfully. To enable this repeatability, managers must get control of commitments and baselines" (p. 12). The top management needs to show firm commitment to learning and development activities in order to "continuously improve the knowledge, skills, motivation, and performance of its workforce" (p.21). As poor relations with immediate supervisor is the primary cause of employee turnover, managers need to be held accountable for ensuring that HR practices are implemented effectively. By providing 
continuous performance feedback, they need to ensure that their team members have the necessary skills and resources to carry out their work and keep their knowledge up-to-date.

Level 3 (Defined: Competency Management): At this level, organisations are able to identify competency-based best practices that most suit the organisational environment and incorporate them as everyday work practices. "These practices are documented and integrated into a common process in which the entire organization is then trained" (p. 13) leading to a professional culture. The workforce capabilities should clearly align with business objectives leading to distinct competitive advantage.

At this level, an organisation-wide framework of strategic workforce plan and workforce competencies is established. This is further augmented by a learning and development plan to impart the knowledge, skills, and process abilities related to each competency . However, employees will not be able to harness their competencies unless the organisational culture empowers the workforce to make decisions independently without the managers with a command and control mindset breathing down their neck. "When the organization achieves Maturity Level 3, the conditions required for empowerment - competent people, effective processes, and a participatory environment - are established" (p.26).

Level 4 (Predictable: Capability Management): Once the work processes are standardised and institutionalised, the organisations acquire the necessary capability to predict and effectively manage its future performance. Where deviations occur, "the cause needs to be determined and corrective action taken if necessary ... When the organization can characterize the performance of its processes quantitatively, it has profound knowledge that can be used to improve them" (p. 13).

In order to further exploit the capability created by the organisational framework set up in level 3, organisations need to institute formal mentoring programs, establish an atmosphere of trust and increased levels of delegation. When competency-based processes have been institutionalized and teams start working autonomously, managers are free from operational issues and they are better able to focus on strategic issues. "The quantitative management capabilities implemented at Maturity Level 4 provide management with better input for strategic decisions, while encouraging delegation of operational details to people close to the processes" (p. 27). 
Level 5 (Optimizing: Change Management): At this highest level of maturity, the work processes and practices undergo continuous improvement to effectively meet the challenges of constantly changing environment. "Managing technological and process change become standard organizational processes and process improvement throughout the organization becomes perpetual" (p. 13). People are empowered to engage in continuous improvement.

At this level, change management becomes an integral and routine part of organisational life. "At Maturity Level 5, individuals are encouraged to make continuous improvements to their personal work processes by analyzing their work and making necessary process enhancements ... Workforce practices are honed to support a culture of performance excellence" (p. 28). At the same time, organisations need to make sure that in an effort to constantly improve, there is no mis-alignment with organisational objectives. They also need to ensure that best practices are shared and implemented across the entire organisational network rather than being isolated to just some parts.

As Curtis, Hefley \& Miller (2009, p. 13) conclude: "the maturity framework builds an environment in which

- practices can be repeated,

- best practices can be transferred rapidly across groups,

- variations in performing best practices are reduced, and

- practices are continuously improved to enhance their capability".

To ensure success, scalability and sustainability, organisations need to gradually scale up the maturity level built on sound foundation of practices.

\section{Conclusion}

Reiterating the critical importance of 'human agency perspective' in IT projects, Boudreau \& Robey (2005, p. 3) observe that "recent perspectives on organisational change have emphasised human agency, more than technology or structure, to explain empirical outcomes resulting from the use of information technologies in organisations". Technology alone cannot result in organisational transformation. Strategic use, involvement and engagement of 
human resources are essential to convert the potential of technology into practice. Otherwise, technology adoption can lead to unintended and undesirable consequences, as illustrated by the case study organisation in this chapter.

Accordingly, the HR department needs to play a strategic role in realising the full potential of information technology, particularly with respect to its role as a strategic business partner, change agent and employee champion (Haines \& Lafleur, 2008; Voermans \& Veldhoven, 2006). Further reinforcing this view, Dery and Wailes (2005) provide evidence suggesting that HR needs to be actively engaged in the introduction and ongoing functioning of IT systems in their organisations in order to realize their full benefits. 


\section{Case Study: Queensland Health Payroll Crisis}

(Source: Extracted from Thite \& Sandhu, 2014)

Queensland (QLD) Health is the government health department in the state of Queensland, Australia and is entrusted with maintaining and promoting the health and well-being of over 4.5 million Queenslanders (see https://www.health.qld.gov.au/ ). It is one of the largest employers in Australia with over 75,000 full-time employees, spread over 300 worksites. The organization has a complex system of employment terms and conditions governed by 12 awards and 6 industrial agreements which provide for over 200 different allowances and up to 24,000 different combinations of pay.

Due to the discontinuation of the old payroll system and to meet its growing needs, QLD Health decided to acquire a new payroll system through the government's IT shared services provider, CorpTech. The best available payroll product and technology vendor (IBM) were selected for the purpose in 2008 . Few months later, it was revealed that 'the size, complexity and scope of this phase of the program had been severely underestimated' resulting in 47 change requests from IBM. Consequently, 'the system implementation was over 18 months after the scheduled Go-Live date and approximately $300 \%$ over the original cost budget'. When the new payroll system went live in March, 2010, QLD Health experienced significant payroll errors resulting in staff being overpaid, underpaid, or not paid at all.

The Auditor-General of Queensland (AGQ, 2010a), highlighted several key reasons for the failure of the new payroll system as below:

- Under-estimation of project scope \& complexity that failed to recognise the organisational complexity leading to very little customisation

- Poor governance and conflict between key agencies (QLD Health, CorpTech and IBM) on roles and responsibilities

- Poor user involvement \& buy-in

- Ill-defined project scope, resulting in 'project creep' (major changes in the middle of the project)

- Poor project management

- Mad rush to Go Live

- Poor risk management 
An independent review identified ongoing people, process and technology issues and recommended the need for comprehensive support for subsequent projects in the form of "change management, training planning and execution to support staff and minimize negative impacts, create consistent and broadly disseminated communication, and effective, targeted training and education". The case study confirms the central message of the chapter that it is not technology per se but the underlying social and organizational processes that mainly derail the implementation and cause the failure to realize the intended benefits.

\section{Case Study Questions}

1. What are the key lessons to be learnt from this case study?

2. Using the SDLC framework, identify the key issues and challenges

3. Explain how the case study organisation can move from level 1 to level 5 as per the People Capability Maturity Model (P-CMM). 


\section{Debate: Should Organisations Outsource Talent?}

\section{Introduction}

Outsourcing refers to letting an outside vendor/ third party carry on the functions instead of doing in-house. It can be on-site (on the company premises), off-site (away from company premises), near shore (near company premises) or off shore (in another country). With increasing globalisation and global competitiveness, organisations around the world are under constant pressure to produce "more with less". With disruptive technologies almost destroying old industries and skills and in the process creating new industries, the human resource management practitioners are similarly under pressure to constantly upgrade the skill profile of their workforce to make it most up-to-date, competent and cost-effective.

\section{Arguments in Favour}

- The main drivers for outsourcing are operating efficiencies or cost reduction; access to specialist expertise; greater process control and risk reduction. Most importantly, for workforce management, it can be a key strategic enabler if planned and implemented strategically.

- Today, talent sourcing has become global with organisations scouting around the world for best talent and at the most competitive price. One can see doctors, nurses, IT managers, software developers, engineers etc. being recruited from a global pool of talent.

- An innovative economy demands state of the art skills that are rapidly changing. This is true particularly in science, technology, engineering and mathematics (STEM) areas. With booming demand for but declining supply of STEM talent due to ageing population etc., emerging economies, such as India and China have emerged as key source countries for STEM talent.

- Today, strategic partnerships and alliances with trust worthy and proven vendors/suppliers across the globe are not only beneficial but imperative to compete.

\section{Arguments Against}

- There are serious negative repercussions of outsourcing talent, such as long-term negative effects on costs, employee morale, and loss of organisational knowledge. If not managed strategically, costs can spiral out of control, employee morale can sink and precious organisational knowledge lost.

- Talent management is indeed a core competency for most high-technology 


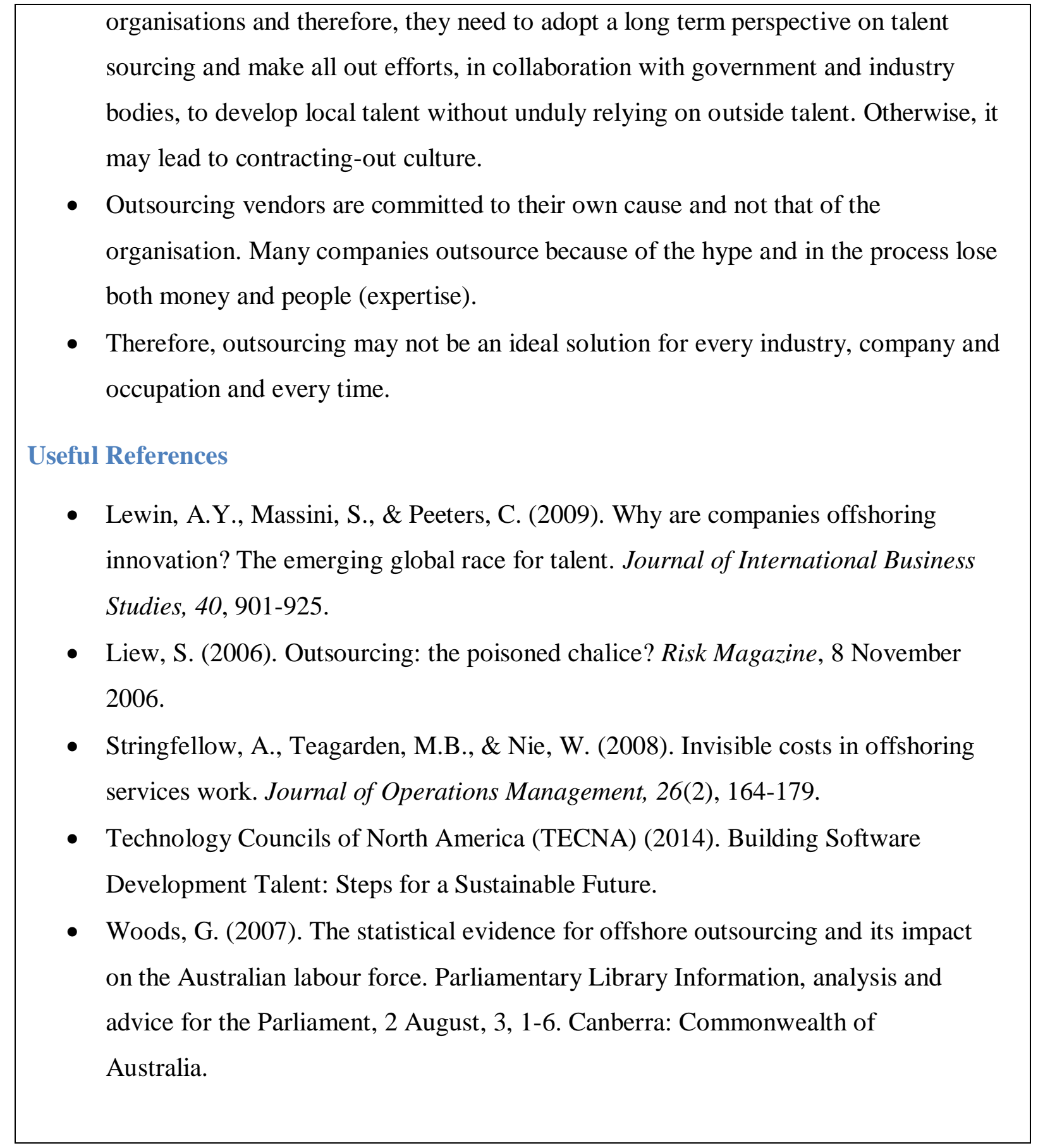




\section{Video Learning Resources}

- What is Project Management? Training Video: https://www.youtube.com/watch?v=9LSnINglkQA

- Software Development Life Cycle (SDLC) - Detailed Explanation: https://www.youtube.com/watch?v=G-6qDY8UltU

- CIS 121 - System Development Life Cycle:

https://www.youtube.com/watch?v=mH-Nc5kvyQQ

- SDLC - Software Development Life Cycle Explained for Beginners | Business Analyst Training: https://www.youtube.com/watch?v=eiXS9dyItnY

- Software Development Life Cycle - simplified:

https://www.youtube.com/watch?v=DRDD7UWX2y4

\section{References}

Arnold, R. D. \& Wade, J. P. (2015). A definition of systems thinking: A systems approach. Procedia Computer Science, 44, 669-678.

Boudreau, M \& Robey, D. (2005). Enacting integrated information technology: A human agency perspective. Organization Science, 16(1): 3-18.

Brock, J. Saleh, T. \& Iyer, S. (2015). Large-scale IT projects: From nightmare to value creation. Bcg.perspectives. Boston Consulting Group. https://www.bcgperspectives.com/content/articles/technology-businesstransformation-technology-organization-large-scale-it-projects/

Curtis, B., Hefley, B. \& Miller, S. (2009). People Capability Maturity Model (P-CMM) Version 2.0, Second edition. Software Engineering Institute, Carnegie Mellon. Technical Report CMU/SEI-2009-TR-003, ESC-TR-2009-003. https://www.sei.cmu.edu/reports/09tr003.pdf

Dery, K and Wailes, W. (2005). Necessary but not sufficient: ERPs and strategic HRM. Strategic Change, 14: 265-272.

Green, A. (2017) Job description of an HRIS manager. https://careertrend.com/about6711738-job-description-hris-manager.html

Hastie, S. \& Wojewoda, S. (2015). Standish Group 2015 Chaos Report: Q\&A with Jennifer Lynch. InfoQ. https://www.infoq.com/articles/standish-chaos-2015 
Hsu, J. S., Liang, T. P., Wu, S. P. J., Klein, G. \& Jiang, J. J. (2011). Promoting the integration of users and developers to achieve a collective mind through the screening of information systems projects. International Journal of Project Management, 29, 514524.

Kim, D. H. (1999). Introduction to systems thinking. Innovations in Management Series. Waltham, MA: Pegasus Communications.

Haines, V. Y. \& Lafleur, G. (2008). Information technology usage and human resource roles and effectiveness. Human Resource Management, 47(3): 525-540.

Martinsons, M. G. \& Chong, P. K. C. (1999). The influence of human factors and specialist involvement on information systems success. Human Relations, 52(1), 123-152.

McConell, S. (1996). Rapid development: Taming wild software schedules. Redmond: Microsoft Press.

Rivera, M. A. (2009). A semi-charmed life: Mike's Journey: The software engineer. http://michang05.blogspot.com.au/2009/05/software-engineer.html

Sankaran, S., Haslett, T., \& Sheffield, J. (2010). Systems thinking approaches to address complex issues in project management. Paper presented at PMI® Global Congress 2010_Asia Pacific, Melbourne, Victoria, Australia. Newtown Square, PA: Project Management Institute. https://www.pmi.org/learning/library/systems-thinking-softmethodology-issues-6912

Senge, P. (1990). The Fifth Discipline, the Art and Practice of the Learning Organization. New York, NY: Doubleday/Currency.

Squires, A., Wade, J., Dominick, P., \& Gelosh, D. (2011). Building a Competency Taxonomy to Guide Experience Acceleration of Lead Program Systems Engineers. In 9th Annual Conference on Systems Engineering Research (CSER) (pp. 1-10). Redondo beach, CA

Thite, M. \& Sandhu, K. (2014). 'Where is my pay? Critical success factors of a payroll system - A system life cycle approach'. Australasian Journal of Information Systems. $18(2), 149-164$.

Standish Group (2014). Law of the Five Deadly Sins. The Standish Group International Inc. https://res.infoq.com/articles/standish-chaos-015/en/resources/5Deadly\%20sins_20145.pdf

Voermans, M. \& Veldhoven, M. (2006). Attitude towards E-HRM: an empirical study at Philips. Personnel Review, 36(6): 887-902.

Vrhoves, S. L. R., Hovelja, T., Vavpotic, D. \& Krisper, M. (2015). Diagnosong organisational risks in software projects: Stakeholder resistance. International Journal of Project Management, 33, 1262-1273. 
Table 3.1: Emotional Maturity \& 5 Deadly Sins of Project Management

\section{Definition of Emotional Maturity:}

"Emotional maturity" is the ability and capacity to perceive, assess, manage, and direct the emotions and actions of the project stakeholders. It is the ability to identify and remove unnecessary requirements, as well as the aptitude to deliver bad news and accept critical feedback. It is the skill to recognize and deal with the Five Deadly Sins of project management, which are overambition, arrogance, ignorance, abstinence, and fraudulence".

\section{“Five Deadly Sins" of Project Management:}

- Overambition: a strong desire to execute a significant project to gain fame, fortune, or power through the impact of overreaching goals.

- Arrogance: the unwarranted, overbearing pride evidenced by a superior manner toward superiors, peers, and inferiors.

- Ignorance: the condition of being unaware, uninformed, uneducated, and/or unsuspecting about the project and stakeholder goals, directions, details, issues, and opportunities. This condition is often coupled with apathy.

- Abstinence: the act or practice of refraining from participation in and contribution to the project.

- Fraudulence: an action intended to deceive; it is deliberate trickery intended to gain an advantage or to avoid confrontation.

Source: Adapted from Standish Group (2014) 


\section{Figure 3.1: System Development Life Cycle}

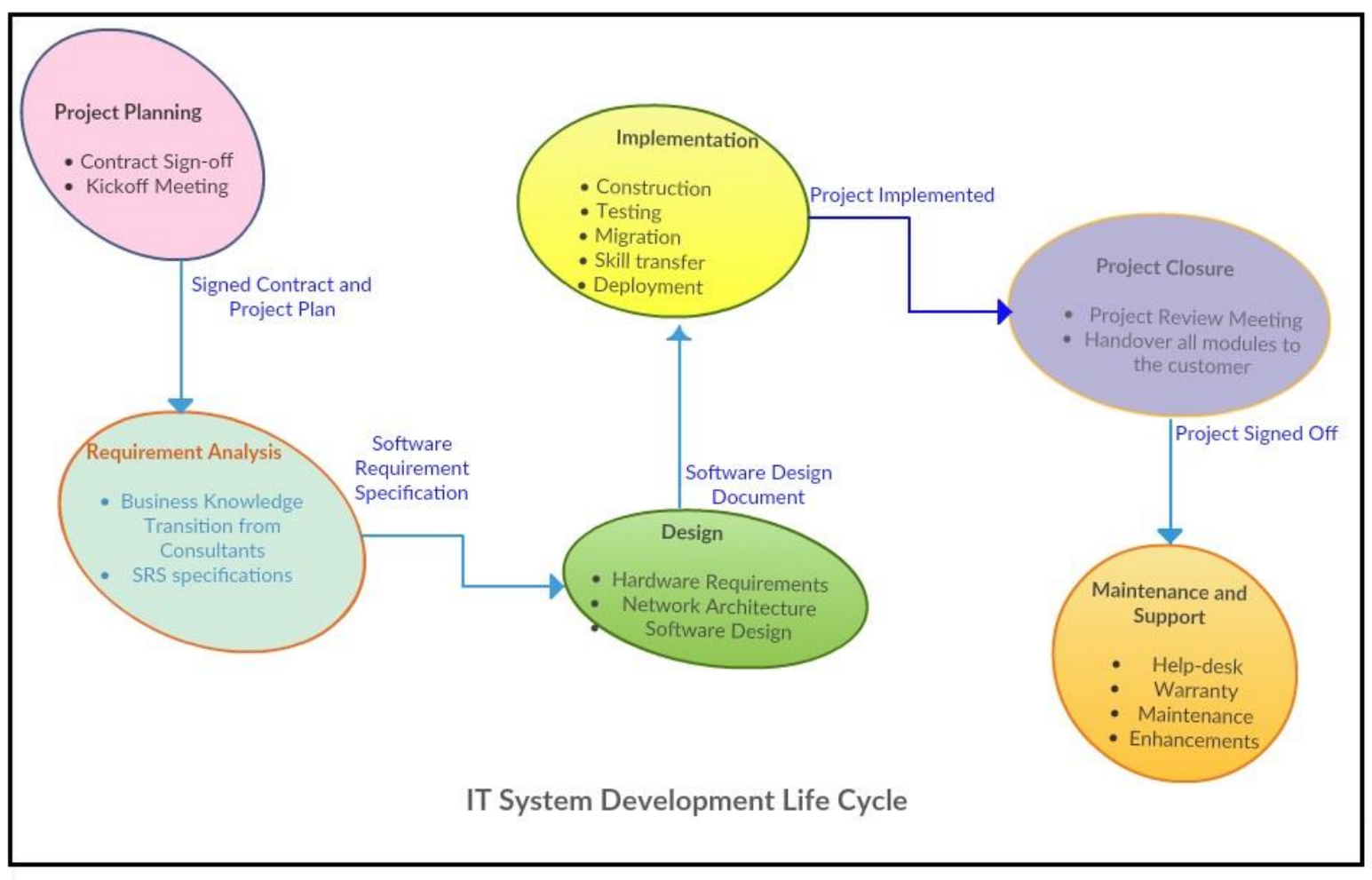

Source: Prepared by NMK Bhatta 
Figure 3.2: Key Success Factors in the Systems Development Life Cycle Source: Thite \& Sandhu (2014)

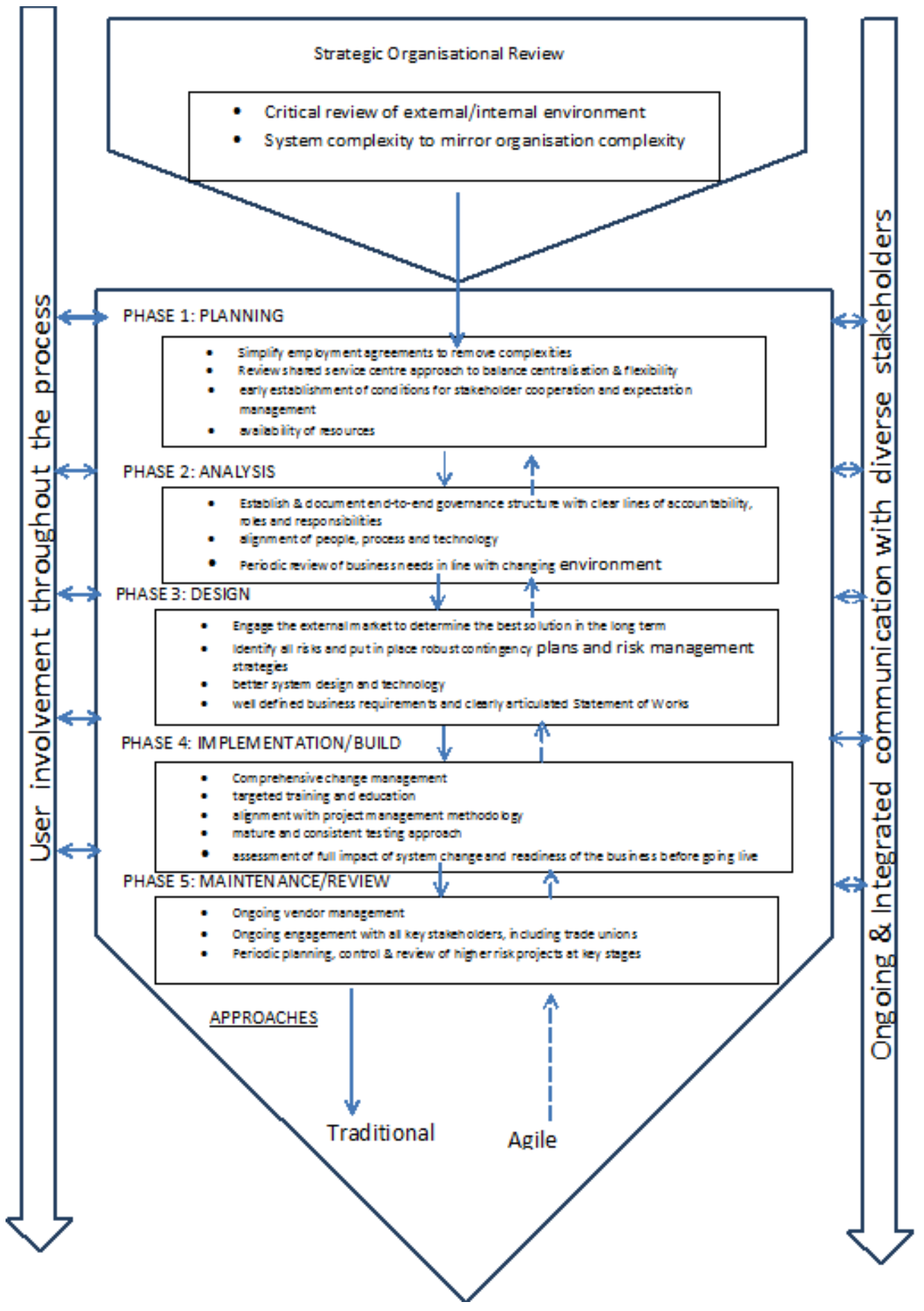


Figure 3.3 People Capability Maturity Model (P-CMM)

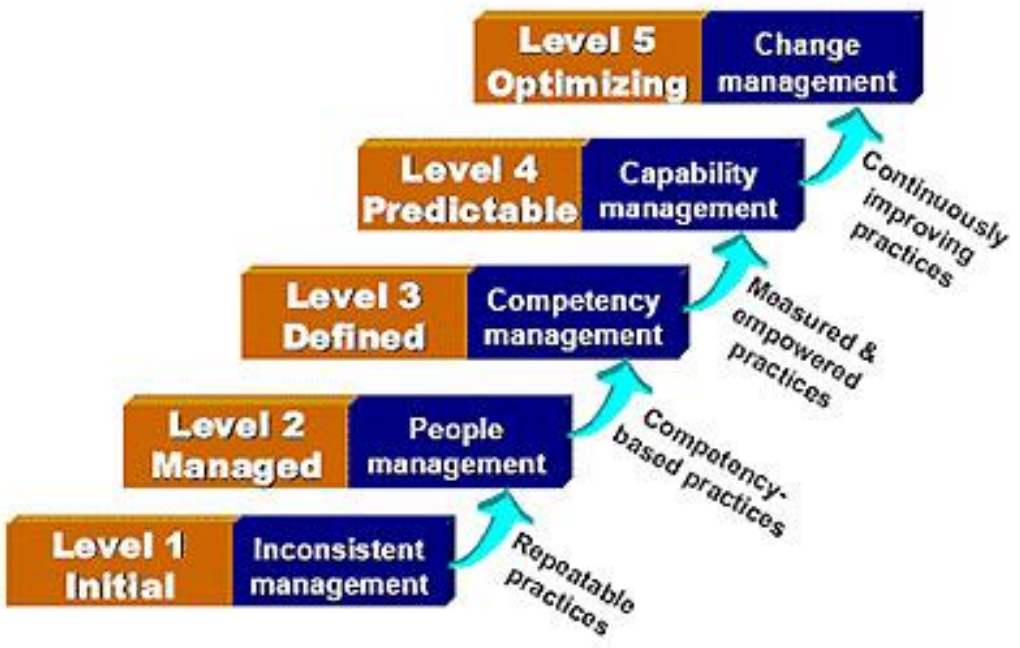

Source: Curtis, Hefley \& Miller (2009, p. 18). Reproduced with Permission from Carnegie Mellon University 